\title{
Hemispheric Asymmetry in Visuotopic Posterior Parietal Cortex Emerges with Visual Short-Term Memory Load
}

\author{
Summer L. Sheremata, ${ }^{1,3}$ Katherine C. Bettencourt, ${ }^{1,3}$ and David C. Somers ${ }^{1,2,3}$ \\ ${ }^{1}$ Department of Psychology, and ${ }^{2}$ Program in Neuroscience, Boston University, Boston, Massachusetts 02215, and ${ }^{3}$ Athinoula Martinos Center for \\ Biomedical Imaging, Charlestown, Massachusetts 02129
}

\begin{abstract}
Visual short-term memory (VSTM) briefly maintains a limited sampling from the visual world. Activity in the intraparietal sulcus (IPS) tightly correlates with the number of items stored in VSTM. This activity may occur in or near to multiple distinct visuotopically mapped cortical areas that have been identified in IPS. To understand the topographic and spatial properties of VSTM, we investigated VSTM activity in visuotopic IPS regions using functional magnetic resonance imaging. VSTM drove areas IPS0 -2, but largely spared IPS3-4. Under visual stimulation, these areas in both hemispheres code the contralateral visual hemifield. In contrast to the hemispheric symmetry observed with visual stimulation, an asymmetry emerged during VSTM with increasing memory load. The left hemisphere exhibited load-dependent activity only for contralateral memory items; right hemisphere activity reflected VSTM load regardless of visual-field location. Our findings demonstrate that VSTM induces a switch in spatial representation in right hemisphere IPS from contralateral to full-field coding. The load dependence of right hemisphere effects argues that memory-dependent and/or attention-dependent processes drive this change in spatial processing. This offers a novel means for investigating spatial-processing impairments in hemispatial neglect.
\end{abstract}

\section{Introduction}

Visual short-term memory (VSTM) refers to the encoding, maintenance, and retrieval of visually presented stimuli that are difficult to name and unlikely to be held in long-term memory (Phillips, 1974). Although our subjective visual experience suggests an effortless ability to encode all of the information before us, VSTM capacity is limited to only three or four objects (Luck and Vogel, 1997). Human neuroimaging studies have demonstrated that activity in the intraparietal sulcus (IPS) closely reflects the number of items held in VSTM (Todd and Marois, 2004; Vogel and Machizawa, 2004; Xu and Chun, 2006; Xu, 2007; Harrison et al., 2010); however, the nature of spatial processing during VSTM is unresolved.

The medial bank of human IPS contains at least five areas, as follows: IPS0 (also known as V7); IPS1; IPS2; IPS3; and IPS4 with visuotopic maps (Tootell et al., 1998; Sereno et al., 2001; Schluppeck et al., 2005; Silver et al., 2005; Swisher et al., 2007; Konen and Kastner, 2008a; Silver and Kastner, 2009). Research has only begun to delineate the specific functions of what may be close to a dozen specific cortical areas in IPS (Astafiev et al., 2003; Grefkes et al., 2004; Orban et al., 2006; Konen and Kastner, 2008a,b).

Each hemisphere of IPS0 - 4 maps the contralateral visual field in a highly symmetric manner. The hemispheric symmetry of topographic maps in IPS sharply contrasts with findings from

Received May 26, 2010; revised July 22, 2010; accepted July 28, 2010.

This work is supported by the National Science Foundation Grant BCS-0726061, the National Center for Research Resources Grant P41RR14075, and the Mental IIIness and Neuroscience Discovery Institute. We thank Jascha Swisher, Mark Halko, and Lingqiang Kong for their helpful feedback and editorial assistance.

Correspondence should be addressed to Summer L. Sheremata, Department of Psychology, Boston University, 2 Cummington Street, \#209, Boston, MA 02215. E-mail: sheremat@bu.edu.

D0I:10.1523/JNEUROSCI.2689-10.2010

Copyright $\odot 2010$ the authors $\quad 0270-6474 / 10 / 3012581-08 \$ 15.00 / 0$ hemispatial neglect syndrome. Patients suffering from hemispatial neglect exhibit severe deficits in contralesional attentional processing predominantly after damage to right hemisphere parietotemporal and/or frontal lobes (Bisiach and Luzzatti, 1978; Heilman and Van Den Abell, 1980; Mesulam, 1981; Vallar and Perani, 1986; Mesulam, 1999; Pouget and Driver, 2000; Doricchi et al., 2008). Although acute, structural damage in hemispatial neglect is normally found ventral to topographic regions of the posterior parietal cortex (PPC), a seminal study (Corbetta et al., 2005) has demonstrated that activity in dorsal PPC regions diminishes during the acute phase of neglect and normalizes with the amelioration of neglect symptoms. These observations suggest that hemispatial neglect emerges from damage to a network including visuotopically mapped IPS (Doricchi et al., 2008). Several studies have investigated the contralateral bias during visual mapping and visual attention (Sereno et al., 2001; Silver et al., 2005; Swisher et al., 2007; Shulman et al., 2010; Szczepanski et al., 2010), but there has not yet been a systematic study of spatial processing in IPS during VSTM. Moreover, at present, we have few insights as to how to study the neglect network in normal healthy human subjects.

Here, we mapped the spatial and functional relationships between VSTM activation and the five visually driven visuotopic cortical areas IPS0-4 (Swisher et al., 2007) using functional magnetic resonance imaging ( $\mathrm{fMRI}$ ). As visuotopic IPS areas are relatively small and reported Talairach coordinates of VSTM IPS activation show considerable variability, we first investigated the spatial overlap of visuotopic maps and areas subserving VSTM within individual subjects. Then, we investigated whether contralateral bias, characteristic of visuotopic maps, is also found for VSTM representations in the parietal cortex. 


\section{Materials and Methods}

Subjects. Nine healthy subjects (4 female, 5 male) with normal or corrected-to-normal vision gave informed written consent to participate in the study, which was approved by the Institutional Review Boards of Boston University and Partners Healthcare. Subjects were recruited from the Boston University community. All subjects were right handed and were between the ages of 23 and 31 years. One author (S.L.S.) served as a subject.

Visual stimuli and experimental paradigm. Visual stimuli were driven by a Macintosh MacBookPro using the Vision Egg software package (Straw, 2008) and were presented using a liquid crystal display projector illuminating a screen within the scanner bore. Subjects viewed the rearprojection screen through a mirror angled at $\sim 45^{\circ}$. The screen extended across a visual angle of $\sim 12^{\circ}$ radius horizontally and $\sim 9^{\circ}$ radius vertically. Subjects fixated a centrally located cross, while eight colored bars oriented vertically or horizontally were presented to the left visual field and eight colored bars were presented to the right visual field (see Fig. 1). The total number of bars per hemifield was kept constant across all trials, but in a given block of trials one, three, or six of the bars were memory targets, whereas the others were distractors. Targets were distinguished from the distractors by color (blue/red, counterbalanced across subjects). Subjects were asked to remember the orientation (horizontal or vertical) of each target. Each bar subtended $0.7^{\circ} \times 0.23^{\circ}$ of the visual angle. The memory sample was presented for $100 \mathrm{~ms}$. After a memory delay of 900 $\mathrm{ms}$, a memory probe was presented for $2000 \mathrm{~ms}$. Trials were $6 \mathrm{~s}$ long, with a $3000 \mathrm{~ms}$ fixation period at the end of each trial. Subjects could respond during the memory probe or during the first $1000 \mathrm{~ms}$ of the post-probe period. In half of the trials, all the bars in the probe had the same orientation as in the memory sample. In the other half of the trials, one of the target bars changed from horizontal to vertical or from vertical to horizontal. Subjects were then asked to report, via button press, whether or not the orientation of the probe target bars matched those of the memory target bars. In the remember-left conditions, all targets were presented left of the fixation cross, whereas the other stimuli were distractors. In the remember-right conditions, all targets were presented to the right. In the remember-full-screen condition, the targets appeared either on the left or the right side of the screen with equal probability. The targets were restricted to a single hemifield in each trial to control for memory capacity across the conditions, as prior studies have revealed greater capacity when targets are spread across both hemifields (Alvarez and Cavanagh, 2005; Delvenne, 2005). Three of the subjects switched their response hand from run to run, whereas six subjects used their right hand exclusively. During passive-viewing trials, subjects were asked to simply press a key when the second set of stimuli appeared.

Subjects' performance for VSTM was measured in terms of capacity. Although multiple estimates of K have been reported, we used Cowan's K (Cowan, 2001). Cowan's K is defined as follows:

$$
\mathrm{K}=\mathrm{SS} \times(\mathrm{HR}-\mathrm{FA})
$$

where SS is the set size, HR is the hit rate, and FA is the false-alarm rate. $\mathrm{K}$ traditionally increases with set size until maximum capacity is reached and then plateaus at larger set sizes. An ANOVA was performed to measure the effects of set size and stimulus location.

Data acquisition. Data were acquired from a 3T Siemens Tim Trio magnetic resonance imager located at the Martinos Center for Biomedical Imaging at Massachusetts General Hospital, Charlestown, MA. Each subject participated in three critical types of scans across multiple sessions: high-resolution structural MRI, visuotopic mapping fMRI, and VSTM fMRI. In an initial session, high-resolution $(1.0 \times 1.0 \times 1.3 \mathrm{~mm})$ structural images were obtained for surface reconstruction, using a 12 channel birdcage head coil. Computer representations of each cortical hemispheric surface were unfolded and flattened using the Freesurfer software package (Dale et al., 1999; Fischl et al., 1999, 2001). Functional scans were acquired using automated motion correction performed by prospective acquisition correction (Thesen et al., 2000). Before functional imaging for each session, T1-weighted EPI images were acquired using the same slice prescription as the functional scans to allow for registration of the data from each session to a subject's high-resolution anatomical images. Visuotopic visual field representations of polar angle and eccentricity were mapped for each subject. T2*-weighted, gradientecho, echo-planar images [repetition time $(\mathrm{TR})=2 \mathrm{~s}$; echo time $=30$ $\mathrm{ms}$; voxel size $=2.8-3.3 \times 2.8-3.3 \times 3.0 \mathrm{~mm}]$ were collected from between 28 and 32 slices covering occipital, parietal, and posterior temporal cortices (scan duration, $8 \mathrm{~min}, 32 \mathrm{~s}$ ). Functional scans measured changes in the intrinsic blood oxygenation level-dependent (BOLD) contrast. Cortical representations of eccentricity and polar angle were mapped with flashing checker-board stimuli in separate runs and were combined using standard techniques (Engel et al., 1994; Sereno et al., 1995; Swisher et al., 2007) with parameters optimized to reveal maps in the parietal cortex. An $F$ test was performed for each voxel to test for a difference between the power at the stimulus frequency and the power of the other frequencies in the time series. For the polar mapping, a wedge was created by contrasting a color-reversing checkerboard against a black background. Subjects were asked to maintain fixation while the wedge rotated around a central fixation point. The polar angle wedge had an arc of $72^{\circ}$, flashed at $4 \mathrm{~Hz}$, had a sweep period of $42.67 \mathrm{~s}$, and swept out 12 cycles per run (for a more detailed description of the visuotopic-mapping methods, see Swisher et al., 2007). Visual stimuli were projected into the patient chamber onto a rear-projection screen (Da-Plex, Da-Lite Screen) and viewed via an adjustable mirror.

In the VSTM study, stimulus location (remember-left, rememberright, and remember-full-screen) and VSTM load (set sizes 1, 3, and 6) varied by block. In addition, subjects performed a single block of passive viewing per run, and block order was randomized within runs and counter-balanced across runs. Each subject performed eight runs, each lasting $7 \mathrm{~min}$ and $12 \mathrm{~s}$, which consisted of $1040 \mathrm{~s}$ blocks as well as $16 \mathrm{~s}$ of blank fixation both before the first block and after the last block. Each block consisted of a $4 \mathrm{~s}$ cue, which indicated the set size and location of the target stimuli, followed by six trials each lasting $6 \mathrm{~s}$. The $40 \mathrm{~s}$ blocks were used to allow subjects to maintain stable attentional and cognitive sets. A total of 32 slices oriented parallel to the calcarine sulcus were collected $(\mathrm{TR}=2 \mathrm{~s}$; voxels $=3.125 \times 3.125 \times 3 \mathrm{~mm})$ using a bilateral quadrature-phase surface coil placed posterior to the occipital pole.

Data analysis. Intensity normalization was performed before signal averaging (Dale et al., 1999; Fischl et al., 1999). Single-subject VSTM fMRI data were analyzed voxel by voxel using a general linear model that included a predictor for each condition (e.g., each location-set size pairing and passive viewing). A $t$ test was performed on each voxel to compare activation differences between conditions, and significance values were projected onto flattened cortical hemisphere representations. For the voxel-by-voxel analysis, the BOLD signal was modeled as a linear time-invariant system; a $\gamma$ response function was assumed or each stimulus condition, with a delay of $\delta=2.25 \mathrm{~s}$ and a decay time constant of $\tau=1.25$. An estimated response was generated by convolving the response function with the stimulus time course (i.e., each block) and minimizing the residual error (FS-FAST, Cortech). Random-effects group analysis was performed using surface-based averaging techniques (Fischl et al., 1999). For the region of interest (ROI) analysis, the percentage signal change data were extracted (from all time points for a block) and averaged by condition, across runs, to construct time-course data for all voxels with a functionally defined ROI. The percentage signal change measure is relative to the average activation level during the fixation condition. Contralateral biases (see Fig. 6) were estimated at each set size for each ROI using the following formula:

$\left(\right.$ Contralateral $\left._{\text {set size }}-\mathrm{Ipsilateral}_{\text {set size }}\right) /\left(\right.$ Contralateral $\left._{\text {set size }}+\operatorname{Ipsilateral}_{\text {set size }}\right)$

VSTM biases were compared across hemispheres using a paired $t$ test.

Visuotopic IPS ROIs IPS0 (also known as V7), IPS1, IPS2, IPS3, and IPS4 were determined using polar angle retinotopy scan data and techniques reported previously (Swisher et al., 2007). For the phase angle analysis of Figure 6, we created binary masks for IPS0 -2 using only those voxels significantly activated $(p<0.05)$ during visuotopic mapping. For each region, we then extracted the preferred phase angle on a voxel-byvoxel basis. Data were summed across subjects and represented using a single polar histogram.

Eye position monitoring. Two subjects returned to perform VSTM during tracking of eye position. The stimuli and procedures for the eye- 
tracking control were the same as for the task described above except for the following changes. A single set size of 3 was used because it approximated the subjects' VSTM capacity. The time before stimulus onset of each trial was reduced, resulting in nine instead of six trials per block without affecting the trial structure. Finally, subjects performed between six and eight runs with each run lasting $5 \mathrm{~min}$ and $36 \mathrm{~s}$. The ISCAN fMRI Remote Eye Tracking Laboratory (ISCAN) recorded subjects' eye positions during scanning. A video camera mounted at the rear of the bore imaged a reflection of the eye via a mirror angled at $45^{\circ}$ using an infrared light-emitting diode illuminator mounted on the head coil. Eye position was sampled at a rate of $120 \mathrm{~Hz}$ and was processed by the ISCAN RK726PCI high-resolution pupil/corneal reflection tracker, located outside of the shielded MRI chamber. Before beginning the task, subject eye position was calibrated to determine both the position of the eye and the size of the screen in terms of the eye-tracking software. In addition, eye position was recorded during various spatially offset fixations at the beginning and end of each run to control for eye position drifts. The eye position data were temporally smoothed by a boxcar filter ( \pm 4 time points) to reduce artifacts caused by blinks and microsaccades. Eye position in each block was recorded and normalized to the median eye position for the two fixation periods for each run and then binned in steps of $0.75^{\circ}$. Each subject's eye-position data were then compared between remember-left and remember-right conditions using a Mann-Whitney test.

\section{Results}

Each of nine subjects participated in a minimum of three sets of scans across multiple sessions. First, high-resolution $(1.0 \times 1.0 \times$ $1.3 \mathrm{~mm}$ ) structural scans were performed to support anatomical reconstruction of the cortical hemisphere surfaces. Retinotopic mapping fMRI scans were performed to identify visuotopic areas IPS0 - 4 within the PPC of each subject hemisphere (Swisher et al., 2007). Visual short-term memory fMRI scans were performed with varying degrees of memory load and varying visual field location of memory targets.

In VSTM scans, subjects fixated a centrally located cross while eight colored bars oriented vertically or horizontally were presented to the left visual field and eight colored bars were presented to the right visual field (Fig. 1). Because visuotopic IPS areas are sensitive to visual drive (Swisher et al., 2007), the number of visual stimuli was kept constant for all conditions. Subjects were asked to remember the orientation (horizontal or vertical) of a subset of the bars that were distinguished from the distractors by color (red or blue). The number of the targets $(1,3$, or 6$)$ and their location (right visual field or left visual field) varied across blocks. In the remember-left conditions, the targets were presented to the left, whereas all of the stimuli on the right were distractors. In the remember-right conditions, the targets were presented to the right, whereas all of the stimuli on the left were distractors. In the remember-full-screen condition, the targets appeared either on the left or the right side of the screen with equal probability.

\section{VSTM behavioral performance}

VSTM behavioral capacity was assessed according to Cowan's K (see Materials and Methods), a measure of the number of items that can be held simultaneously in short-term memory (Cowan, 2001). For all three target locations (full-screen, right, and left), $\mathrm{K}$ score increased and reached a plateau with increasing set size (Fig. 2 ). These results are consistent with prior behavioral reports (Cowan, 2001; Todd and Marois, 2004; Vogel and Machizawa, 2004; Xu and Chun, 2006). Overall, $\mathrm{K}$ increased from 0.91 at set size 1 , to 1.94 at set size 3 , and to 2.17 at set size 6 (rememberleft $=0.90,1.85,2.00$; remember-right $=0.93,1.87,2.21$; remember-full-screen $=0.92,2.09,2.29)$. A repeated-measures ANOVA revealed a significant effect of set size $\left(F_{(2,16)}=44.07\right.$, $p<0.001)$, which represented a significant difference between set

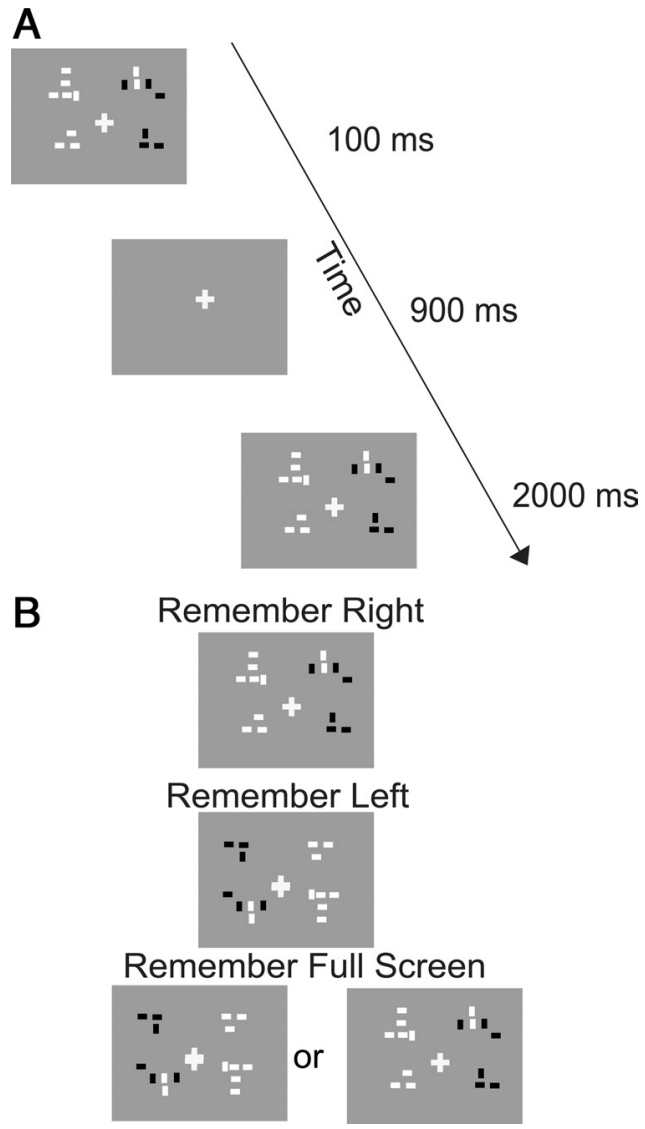

Figure 1. VSTM trial structure. $A$, Subjects encoded a set of briefly presented targets among distractors, and after a delay were asked whether the orientation of any of the targets had changed. During the experiment, targets and distractors were presented in red and blue, respectively (colors randomized between subjects). Here, targets are depicted in black, and distractors in white. $\boldsymbol{B}$, Target location conditions. In trial blocks, targets appeared always in the right visual hemifield (remember-right), always in the left visual hemifield (remember-left), or could appear in either hemifield (remember-full-screen). The number of targets also varied across blocks (set sizes 1,3, and 6), but the total number of stimuli remained constant.

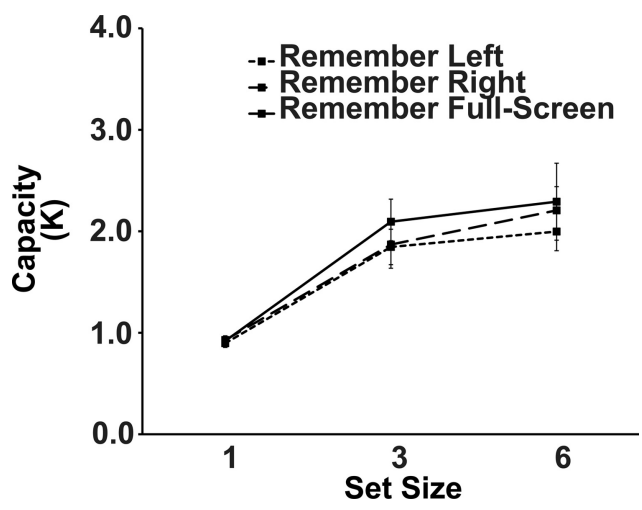

Figure 2. Average VSTM K score for each set size and location. K score plateaus for each memory location, with no significant differences between the locations.

sizes 1 and $3\left(t_{(26)}=9.80, p<0.001\right)$ and set sizes 1 and $6\left(t_{(26)}=\right.$ $8.64, p<0.001)$, but no significant difference between set sizes 3 and 6 . There was neither a significant effect of target location nor an interaction between set size and target location, demonstrating that memory performance was not different across the visual hemifields. 

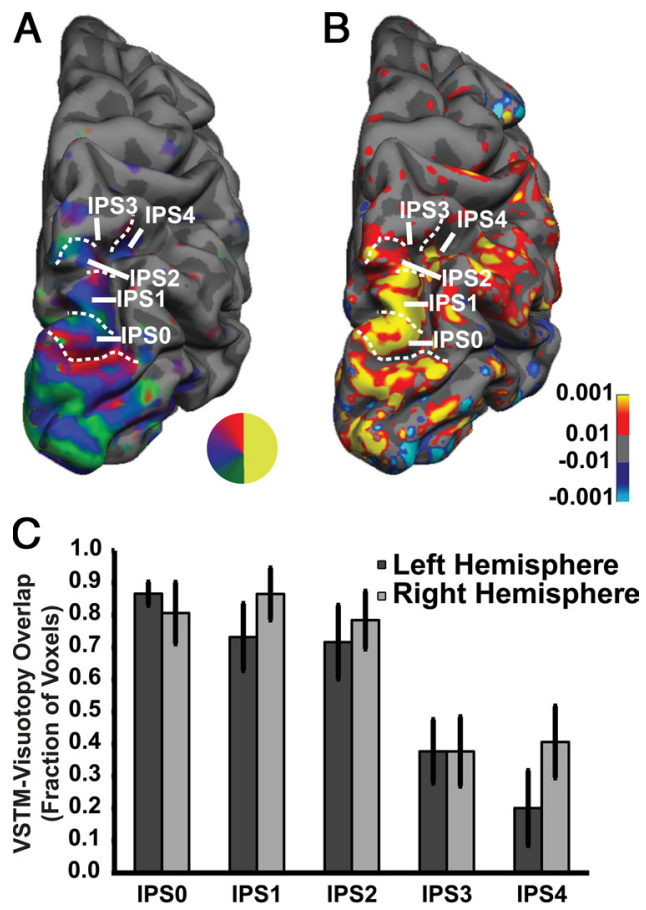

Figure 3. $A, B$, Activation in corresponding regions of the dorsal posterior parietal cortex for visuotopic mapping $(\boldsymbol{A})$ and VSTM $(\boldsymbol{B})$ for a representative subject (remember-full-screen for set sizes 3 and 6 vs fixation). Both activations overlap along the medial bank of the posterior branch of IPS in visuotopically defined areas IPS0/V7, IPS1, and IPS2. VSTM activity continued along the lateral/inferior bank of the anterior branch of IPS, showing decreased overlap with IPS3 and 4, which continued in a relatively more superior/medial bank of the anterior branch of IPS. C, Percentage of voxels in each visuotopic IPS area that exhibit memory load-dependent activation in the VSTM task.

Table 1. Talairach coordinates for the centroids of regions IPSO -4 and lateral anterior IPS

\begin{tabular}{ll}
\hline ROIS & Talairach coordinates \\
\hline IPS0 & $\pm 26,-78,29(6,5,6)$ \\
IPS1 & $\pm 22,-71,41(5,5,6)$ \\
IPS2 & $\pm 18,-63,52(4,6,8)$ \\
IPS3 & $\pm 21,-56,56(4,7,5)$ \\
IPS4 & $\pm 26,-48,55(5,6,7)$ \\
Lateral anterior IPS & $\pm 38,-45,44(2,2,1)$ \\
\hline
\end{tabular}

Regions IPSO -4 were defined by visuotopic mapping, whereas lateral anterior IPS was defined by regions of IPS active during the remember-full-screen condition but was not encompassed by IPSO -4 .

\section{Overlap between VSTM activation and visuotopic IPS}

Visualization of fMRI activation patterns for the remember-fullscreen VSTM data revealed significant overlap of the areas activated by VSTM and visuotopic mapping (Fig. 3a,b). Both visuotopic mapping and VSTM showed a band of activity along the medial bank of IPS. Along the posterior branch of IPS, VSTM activity and visuotopic mapping activated overlapping regions. This region corresponds to visuotopic areas IPS $0-2$. Lateral and anterior to these areas, VSTM activity and visuotopic areas IPS3-4 showed reduced overlap, with the VSTM activity continuing along the lateral/inferior bank of the anterior branch of IPS. In contrast, visuotopic areas of IPS were superior and medial compared with VSTM activity.

To quantify the extent of overlap for each of the regions, we created ROIs for each visuotopic area IPS0 (V7) to IPS4 based on the retinotopic mapping scans (Table 1, Talairach coordinates), and we created VSTM-IPS ROIs for each subject hemisphere by contrasting the remember-full-screen activation for set sizes 3 and 6 with passive viewing and fixation. Then, we created conjunction ROIs by taking only those voxels significantly activated $(p<0.05)$ for both visuotopic mapping and VSTM. We compared the number of voxels activated in these conjunctions compared with the voxels significantly activated by visuotopic mapping alone. The results for left and right hemispheres are shown in the bar graph of Figure 3C. Approximately $80 \%$ overlap was observed in IPSO-2. A repeated-measures ANOVA revealed a significant effect of ROI $\left(F_{(4,32)}=19.530, p<0.001\right)$. This ROI effect matches the effect qualitatively observed in Figure $3 A, B$. Paired comparisons showed less overlap between visuotopic mapping and VSTM in IPS3 -4 than in IPS0 -2 (IPS3 vs IPS0: $t_{(17)}=5.194, p<0.001$; IPS3 vs IPS1: $t_{(17)}=5.172, p<0.001$; IPS3 vs IPS2: $t_{(17)}=5.336, p<$ 0.001 ; IPS4 vs IPS0: $t_{(17)}=6.310, p<0.001$; IPS4 vs IPS1: $t_{(17)}=$ 6.277, $p<0.001$; and IPS4 vs IPS2: $\left.t_{(17)}=4.908, p<0.001\right)$, but there were no significant differences among regions IPS $0-2$ or between IPS3 and IPS4. There was neither a significant effect of hemisphere nor an interaction between hemisphere and ROI demonstrating that in the remember-full-screen condition, the right and left hemispheres exhibited highly symmetric VSTM activation. These results also demonstrate a functional dissociation for VSTM in IPS. Areas recruited in VSTM are strongly coincident with areas IPS0 -2, but largely spare areas IPS3-4, with the overlap primarily in the fundus of IPS. Although one must always be cautious when comparing effects across ROIs, we note that visuotopic areas IPS0 -4 follow the medial bank of IPS, whereas VSTM activation follows the medial bank of posterior IPS and the lateral/inferior bank of anterior IPS. This anatomical distinction also indicates that VSTM activation is coincident with IPS0-2, but largely lateral/inferior to IPS3-4.

\section{Effects of set size and visual hemifield on BOLD signal in IPS}

We analyzed the remember-full-screen, remember-left, and remember-right conditions in both hemispheres for each VSTM set size. For each hemisphere, the conditions were treated as fullscreen, contralateral, and ipsilateral locations. Because there was minimal overlap between VSTM and visuotopic mapping for regions IPS3-4, we restricted further analyses to regions IPS0 -2. A repeated-measures ANOVA revealed a significant main effect of set size $\left(F_{(2,16)}=4.52, p=0.028\right)$. This can be seen qualitatively in Figure $4 A-C$ (remember-full-screen) and quantitatively in Figure $4 D$ (all 3 locations together). BOLD signal mirrored subjects' memory capacity, with a significant difference between set sizes 1 and $3\left(t_{(161)}=6.33, p<0.001\right)$ and set sizes 1 and $6\left(t_{(161)}=\right.$ $6.10, p<0.001)$, but not between set sizes 3 and 6 . These results indicate that visuotopic areas IPS -2 are increasingly recruited as subjects remember a greater number of items, arguing for a functional role during VSTM. The location of activity is similar to prior reports of IPS that did not identify visuotopic areas (Todd and Marois, 2004; Xu and Chun, 2006; Xu, 2007).

To be activated during visuotopic mapping, a visually responsive voxel must show a spatial preference in terms of its population receptive field. If the VSTM BOLD signal in IPSO-2 reflects activity resulting from the same neural populations, these voxels would be expected to show a strong contralateral bias for VSTM representations. A significant main effect of memory stimulus location (i.e., contralateral $>$ ipsilateral) would indicate that during VSTM there is a bias for remembered items presented in the contralateral visual hemifield. Consistent with this hypothesis, the repeated-measures ANOVA revealed a significant main effect of visuotopic location $\left(F_{(2,16)}=3.70, p=0.048\right)$. Paired comparisons showed a significant difference between the contralateral and ipsilateral $\left(t_{(161)}=4.86, p<0.001\right)$ and ipsilateral and fullscreen $\left(t_{(161)}=2.44, p<0.029\right)$ conditions, but no significant 

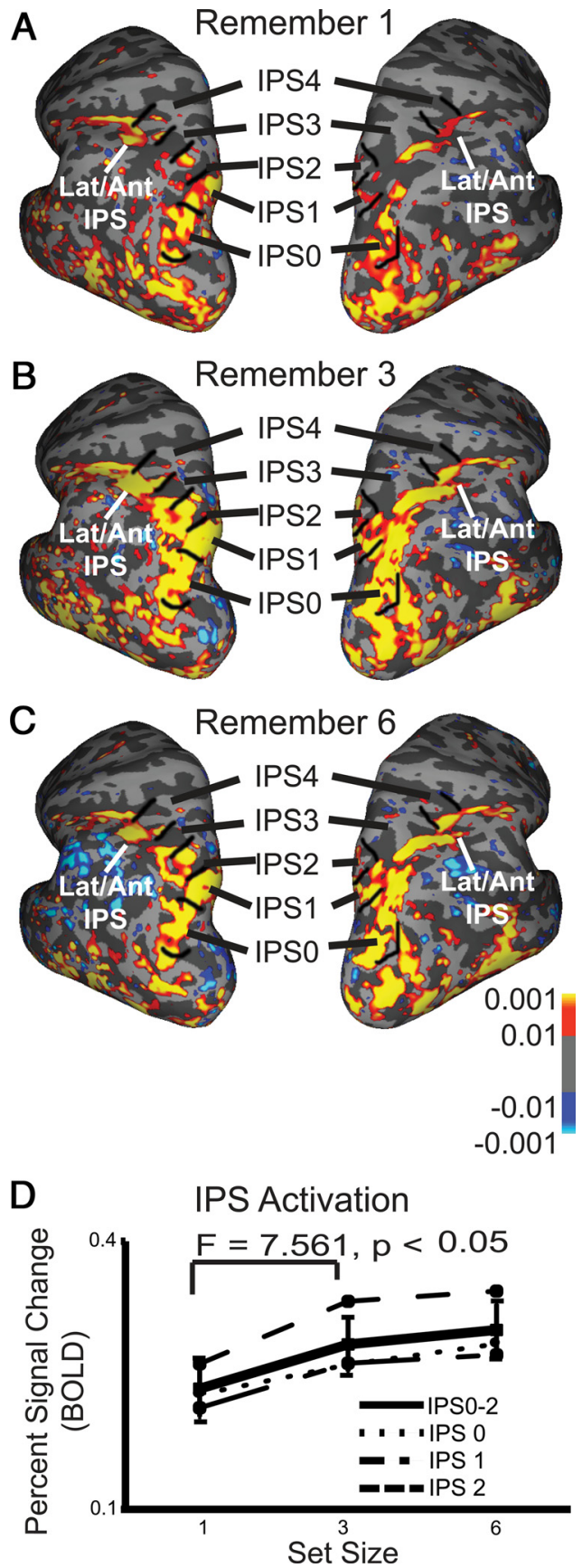

Figure 4. Effects of VSTM set size on IPS BOLD activity. $A-C$, Activation from the rememberfull-screen condition (vs fixation) for a single subject for $1(\boldsymbol{A}), 3(\boldsymbol{B})$, and $6(\boldsymbol{C})$ targets. $\boldsymbol{D}$, BOLD signal averaged across all 3 conditions exhibits a plateau that mirrors the behavioral (K score) performance shown in Figure 2 . The thin lines represent IPSO -2 separately and the thick line reflects their combined ROI. Lat, Lateral; Ant, anterior.

difference between contralateral and full-screen conditions. These results are largely expected, given the demonstrated overlap between VSTM activation and visuotopic maps.

Although there was not an overall effect of hemisphere, there was evidence of hemispheric asymmetry in VSTM processing. There were significant interactions between set size and hemisphere $\left(F_{(2,16)}=3.65, p=0.049\right)$, and visual location and hemisphere $\left(F_{(2,16)}=5.00, p=0.021\right)$. In addition, there was a marginally significant three-way interaction among visual location, hemisphere, and set size $(F=2.38, p=0.07)$.
A

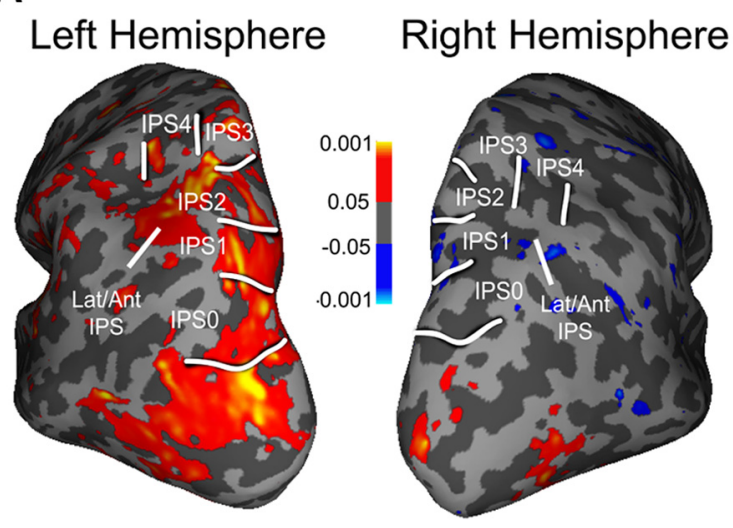

B

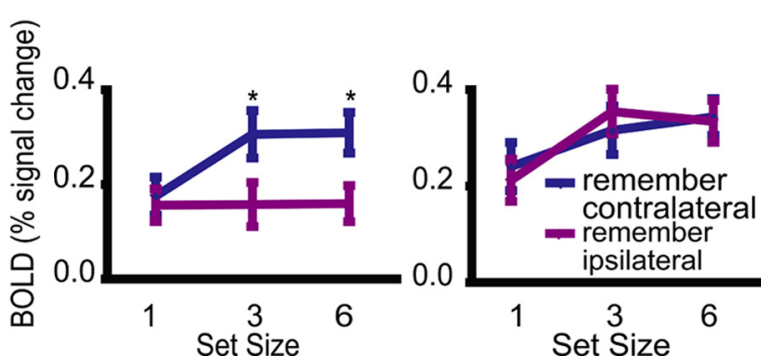

Figure 5. Group average contralateral bias (contralateral $>$ ipsilateral). $\boldsymbol{A}$, Group average of areas showing significantly greater activity for contralateral than ipsilateral locations for set sizes 3 and 6 combined. $\boldsymbol{B}$, Activity for contralateral and ipsilateral conditions for each set size for IPSO -2 in each cortical hemisphere. Lat, Lateral; Ant, anterior.

To better investigate the hemispheric asymmetry, we compared contralateral and ipsilateral responses for the two hemispheres. The group average for the high-load conditions (contralateral vs ipsilateral for set sizes 3 and 6) revealed a dramatic asymmetry (Fig. 5a). Under high-load conditions (set sizes 3 and 6), the left hemisphere exhibited a pattern of strong contralateral bias for VSTM that one would expect for visuotopic areas. In contrast, no contralateral bias was observed for the right hemisphere. Figure $5 B$ displays the activation level for each hemisphere, for each set size and location. The left hemisphere exhibited increasing BOLD signal with increasing set size for contralateral stimuli only (set size 1 vs set size $3, t_{(23)}=3.51, p<$ 0.01 ; set size 1 vs set size $\left.6, t_{(23)}=4.12, p<0.001\right)$; ipsilateral responses were flat across set sizes. In contrast, the right hemisphere exhibited increasing BOLD signal with increasing set size for both contralateral and ipsilateral memory targets (contralateral: set size 1 vs set size $3, t_{(23)}=3.18, p<0.01$; set size 1 vs set size $6, t_{(23)}=4.58, p<0.001$; ipsilateral: set size 1 vs set size $3, t_{(23)}=$ $7.23, p<0.001$; set size 1 vs set size $\left.6, t_{(23)}=4.94, p<0.001\right)$. These results reveal a surprising, load-dependent, hemispheric asymmetry in VSTM processing in which the left hemisphere is strongly biased toward coding contralateral targets, but the right hemisphere robustly codes targets in both hemifields. Control studies ruled out eye position and response hand explanations of this hemispheric asymmetry (supplemental materials, available at www.jneurosci.org as supplemental material).

The combined results present a paradox. Visuotopic IPS shows a strong bias for stimuli in the contralateral visual field for both cortical hemispheres. This hemispheric symmetry contrasts with the asymmetry observed for VSTM activation. To confirm that both results hold for the same ROI voxels, we conducted an 
analysis in which the preferred polar angle during retinotopic mapping scans was extracted for each voxel of each subjects' ROIs. We created binary brain masks for IPS0-2 using only those voxels significantly activated $(p<0.05)$ during visuotopic mapping. The preferred angles were then summed across subjects and plotted on a polar histogram (Fig. 6A). The results demonstrate that each ROI showed a consistent and hemispherically symmetric contralateral bias during visuotopic mapping.

Conversely, analysis of these same voxels during VSTM revealed there was a hemispheric asymmetry in the degree of contralateral bias (Fig. 6B). In the left hemisphere, there was a significant contralateral bias for set sizes 3 and 6 for IPS0 (set size $3, t_{(8)}=3.564, p<0.01$; set size 6 , $t_{(8)}=6.20, p<0.001$ ), IPS1 (set size 3 , $t_{(8)}=2.449, p=0.040$; set size $6, t_{(8)}=$ $3.029, p=0.016$ ), and IPS2 (set size 3, $t_{(8)}=2.351, p=0.047$; set size $6, t_{(8)}=$ $3.744, p<0.01)$. There was no significant effect of stimulus location for the right hemisphere for any ROI at any set size. The contralateral bias between the hemispheres was not different at set size 1, but was significantly greater in the left hemisphere than the right for set sizes $3\left(t_{(26)}=3.31, p<0.01\right)$ and $6\left(t_{(26)}=2.80, p<0.01\right)$. These results confirm that the hemispheric asymmetry for VSTM emerges in IPS voxels that are strongly and symmetrically driven by contralateral visual representations.

In addition to areas IPS $0-2$, the memory task also activated a region lateral and anterior to areas of overlap between VSTM and visuotopic mapping. Although we did not observe visuotopic maps in this region, we cannot rule out the existence of visuotopic maps within this lateral and anterior IPS region (Konen and Kastner, 2008b). We performed an additional repeated-measures ANOVA for this region, referred to as lateral anterior IPS, to investigate effects of set size and stimulus location to characterize the involvement of this region in VSTM. A main effect of set size in this region approached significance $\left(F_{(2,16)}=2.907, p=0.08\right)$ but a significant interaction between set size and hemisphere was observed $\left(F_{(2,16)}=7.077, p<0.01\right)$. Consistent with VSTM activation in visuotopic IPS, paired comparisons demonstrated that this was the result of a significant effect of memory stimulus location for the left hemisphere $\left(t_{(26)}=3.158, p<0.01\right)$, but not for the right hemisphere. Thus, this lateral, anterior IPS activation also exhibited the same hemispheric asymmetry observed in IPSO -2 , but we did not observe the symmetric visuotopic maps of the contralateral field.

\section{Control measurements: eye position monitoring and response hand}

It is difficult to imagine how eye position effects could account for the observed hemispheric asymmetry. Conceivably, subjects could shift their gaze rightward during remember-right trials, thus placing some targets contralateral to both right and left hemispheres, and yet hold central fixation during remember-left trials, resulting in only the right hemisphere receiving contralateral targets. Such an eye position asymmetry seems highly improbable; nevertheless, we examined this with two subjects. Eye

\section{B Contralateral Bias VSTM}
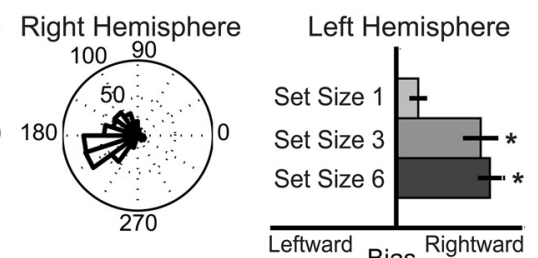

Right Hemisphere
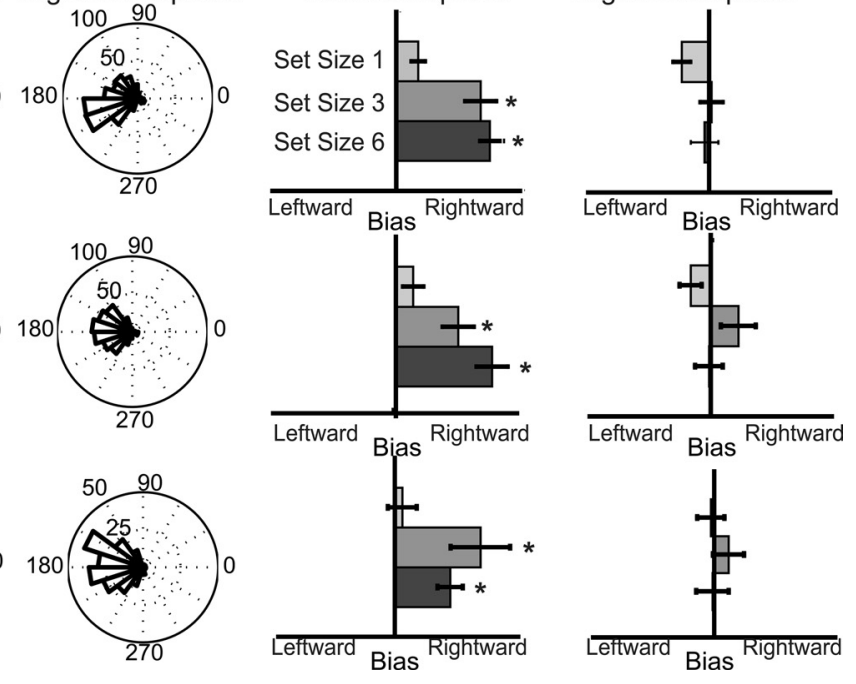

Figure 6. Comparison of contralateral bias during visuotopic mapping and VSTM. $A$, Preferred visual angle for voxels significantly active during visuotopic mapping in regions IPSO -2 for the left and right hemispheres. $\boldsymbol{B}$, Contralateral bias [(contralaterralateral $_{\text {set size }}+$ ipsilateral $_{\text {set size }}$ ] during VSTM for the same ROIs as in $\boldsymbol{A}$.

position was monitored in the scanner while subjects performed remember-left, remember-right, and remember-full-screen conditions at set size 3 . Subjects held fixation well across the conditions with no significant difference between remember-right and remember-left conditions (supplemental Fig. 1, available at www.jneurosci.org as supplemental material). The subjects exhibited the same degree of parietal asymmetry that they did in the full experiments. Thus, eye position effects do not account for our observations.

It is also possible that the hand with which subjects responded influenced the spatial bias, resulting in a contralateral bias for the left but not the right hemisphere. Again, it is hard to conceive how right-handed button presses would influence only the ipsilateral hemisphere; nevertheless, we did investigate this issue. To determine whether subjects' responses influenced the hemispheric spatial biases, we asked a set of our subjects $(n=3)$ to respond with the left hand for half of the runs. There was no interaction among the response hand, hemisphere, and location. Supplemental Figure 2 (available at www.jneurosci.org as supplemental material) displays the hemifield biases of each hemisphere for the subjects who only used their right hand to respond with those who switched hands. If anything, the hemispheric asymmetry is greater in those subjects who switched hands. At higher load, the switched-hand subjects tend toward an ipsilateral bias for the right hemisphere, compared with the strong contralateral bias for the left hemisphere. Thus, neither response hand nor eye position accounts for the hemispheric asymmetry observed in VSTM.

\section{Discussion}

We observed that activation in the human intraparietal sulcus reflected visual short-term memory performance across varying memory loads. As VSTM set size increased, BOLD signal and K score both increased and then reached a plateau. In contrast to previous studies (Todd and Marois, 2004; Xu and Chun, 2006; $\mathrm{Xu}, 2007)$, here the number of visual stimuli were constant across memory loads, confirming that set size effects in IPS are due to memory load, not to visual drive. IPS, the most prominent ana- 
tomical feature within lateral PPC, has been treated by prior VSTM researchers (Todd and Marois, 2004; Vogel and Machizawa, 2004; Xu and Chun, 2006; Xu, 2007; Harrison et al., 2010) as consisting of either a single region or two subregions (e.g., inferior and superior IPS).

Recent studies have demonstrated that the medial/superior bank of IPS alone contains no fewer than six distinct visuotopic areas (Swisher et al., 2007; Konen and Kastner, 2008b; Silver and Kastner, 2009; Szczepanski et al., 2010). The Talairach coordinates that we and a previous study (Swisher et al., 2007) observed for the centroids of IPS1-4 lie within an SD of the Talairach coordinates of the key VSTM activation reported in prior fMRI studies (Todd and Marois, 2004; Xu and Chun, 2006; Xu, 2007; Harrison et al., 2010). A functional dissection of IPS is necessary, and the present study takes an important step forward toward that goal.

A recent study (Xu and Chun, 2006) reported that superior IPS activity better reflects VSTM processing than inferior IPS activity. Taking these independent findings together, one might assume that VSTM should be centered in IPS3-4, the more superior visuotopic IPS areas. However, the present results yield a different picture. Within visuotopically mapped IPS, we observed that VSTM activation overlapped $\sim 80 \%$ ( $72-87 \%$ ) of visuotopic areas IPS0-2, with the VSTM activity shifted slightly lateral/inferior to the IPS areas. In contrast, the overlap in IPS3-4 is only $20-40 \%$. Although stimulus location was not varied at a fine scale, prior work from our laboratory demonstrates that foveal stimuli are represented on the lateral side of the IPS areas (Swisher et al., 2007). Since the fovea is poorly mapped in polar angle mapping (it is activated by all polar angles and thus is a singularity) and since the VSTM task contains perifoveal stimuli, we interpret our findings as supporting a complete overlap in IPS0-2 for VSTM and visuotopic mapping. Our observations suggest a functional distinction between visuotopic IPS areas. IPS0-2 appear to play central roles in VSTM, whereas the roles of IPS3-4, which exhibit much more modest overlap with VSTM activation, are less clear.

Surprisingly, we observed a strong hemispheric asymmetry in the contralateral bias of IPS0 - 2 that emerged as VSTM load increased. Given that IPS $0-2$ contain visuotopic maps of the contralateral visual field and are strongly driven by VSTM load, we expected each cortical hemisphere to exhibit a strong bias for contralateral VSTM targets. Left hemisphere IPS was strongly biased for contralateral targets, but right hemisphere IPS exhibited set size effects for stimuli placed in either visual hemifield. This presents a paradox in that the right hemisphere IPS voxels are strongly biased for contralateral visual stimuli but code shortterm memory targets across the entire visual field.

The results of our visuotopic mapping task strongly reflect visual drive rather than cognitive factors. Attention is always directed to one location, and the stimuli are complex and task irrelevant, minimizing the chance that a subject would try to remember them. In contrast, the VSTM scans kept the visual drive constant across all conditions and varied the memory load, and thus the activation reflects memory processes. In the left hemisphere, the spatial representations of visual percepts and short-term memory representations appear to be consistent; however, in the right hemisphere, we observe a major difference between the sensory and mnemonic spatial representations. Right hemisphere IPS0-2 exhibit contralateral spatial maps for stimuli, but exhibit mnemonic responses equally strongly for stimuli in either visual hemifield. Such hemispheric asymmetries are not found for memory-guided delayed saccade tasks (Sereno et al., 2001; Schluppeck et al., 2005). One possible explanation is that motor preparation, a confound in the delayed saccade task (Barash et al., 1991), may result in different patterns of topography compared with the memory component. Alternatively, the number of remembered stimuli may influence the topography, as we observed this asymmetry only when more than one target was remembered.

This hemispheric asymmetry could potentially be explained by either of two hypotheses regarding right hemisphere IPS. Right IPS, like many cortical areas, might contain two distinct neuronal populations: one population that performs mnemonic spatial indexing of the contralateral field and another population that performs nonspatial memory processes. In contrast, the left hemisphere might contain only contralateral spatial indexing neurons. This dual-population hypothesis implies that the right hemisphere responses should still be greater for contralateral than ipsilateral memory targets; however, our observations do not support this. Alternatively, right IPS could be driven by different sets of inputs that switch the effective spatial representation, depending on which are more strongly activated by the task. This dual-input hypothesis suggests that bottom-up visual stimulation arrives only from the contralateral field, whereas mnemonic inputs arrive from the entire visual field. This hypothesis is consistent with our observations and with single-unit primate studies that have shown that the same neurons can alter their firing patterns depending upon the task demands both in terms of representation of items (Toth and Assad, 2002) and the coordinate system in which objects are encoded (Battaglia-Mayer et al., 2003). These mnemonic inputs might come from ventral parietal and superior temporal regions, the primary loci of lesions underlying hemispatial neglect, or from lateral anterior IPS, which exhibits the same hemispheric asymmetry for VSTM as IPSO-2, but does not exhibit contralaterally biased visuospatial maps.

Hemispatial neglect is a complex syndrome that reflects a disruption in spatial attention in which contralesional neglect occurs almost exclusively after right hemisphere damage. To explain this hemispheric asymmetry, representational models of hemispatial neglect (Heilman and Van Den Abell, 1980; Mesulam, 1981, 1999; Pouget and Driver, 2000) have suggested that the right hemisphere should contain bilateral maps, whereas the left hemisphere should contain only contralateral maps. Prior neuroimaging studies have observed multiple spatial maps in the dorsal parietal attentional network (Sereno et al., 2001; Schluppeck et al., 2005; Silver et al., 2005; Swisher et al., 2007; Konen and Kastner, 2008b); however, these reports have failed to observe significant hemispheric asymmetries in these maps. A recent study reported a modest attentional asymmetry in visuotopic IPS1-2 (Szczepanski et al., 2010), whereas another recent study found a strong attentional asymmetry in ventral parietal cortex, but no asymmetry in the dorsal parietal attentional network (Shulman et al., 2010). Here, using a VSTM task, we observed robust bilateral field representations in right hemisphere IPS0-2 and strongly biased contralateral field representations in left hemisphere IPS0-2, consistent with the predictions of representational models of neglect. We also note that this asymmetry only emerges when subjects try to hold more than one object in short-term memory, and previous studies have not looked at the differences for attending to multiple objects. A seminal study (Corbetta et al., 2005) has previously demonstrated that, although dorsal parietal areas are not typically damaged by strokes causing hemispatial neglect, these areas, including regions of IPS, show greatly reduced activity during the acute phase of neglect. 
As neglect symptoms recede, dorsal parietal activation increases. To explain this phenomenon, we suggest a network model of hemispatial neglect in which temporoparietal damage causes a disruption of inputs to the dorsal parietal regions that explicitly encode for spatial coordinates (Corbetta et al., 2005). Our findings are consistent with such a network model and provide fMRI evidence that hemispatial neglect mechanisms may be studied in normal human subjects. Our work suggests that one key is to use tasks that sufficiently tax subject capacities, such as requiring the coding of multiple stimuli within a visual hemifield.

\section{References}

Alvarez GA, Cavanagh P (2005) Independent resources for attentional tracking in the left and right visual hemifields. Psychol Sci 16:637-643.

Astafiev SV, Shulman GL, Stanley CM, Snyder AZ, Van Essen DC, Corbetta M (2003) Functional organization of human intraparietal and frontal cortex for attending, looking, and pointing. J Neurosci 23:4689-4699.

Barash S, Bracewell RM, Fogassi L, Gnadt JW, Andersen RA (1991) Saccaderelated activity in the lateral intraparietal area. I. Temporal properties; comparison with area 7a. J Neurophysiol 66:1095-1108.

Battaglia-Mayer A, Caminiti R, Lacquaniti F, Zago M (2003) Multiple levels of representation of reaching in the parieto-frontal network. Cereb Cortex 13:1009-1022.

Bisiach E, Luzzatti C (1978) Unilateral neglect of representational space. Cortex 14:129-133.

Corbetta M, Kincade MJ, Lewis C, Snyder AZ, Sapir A (2005) Neural basis and recovery of spatial attention deficits in spatial neglect. Nat Neurosci 8:1603-1610.

Cowan N (2001) The magical number 4 in short-term memory: a reconsideration of mental storage capacity. Behav Brain Sci 24:87-114.

Dale AM, Fischl B, Sereno MI (1999) Cortical surface-based analysis. I. Segmentation and surface reconstruction. Neuroimage 9:179-194.

Delvenne JF (2005) The capacity of visual short-term memory within and between hemifields. Cognition 96:B79-B88.

Doricchi F, Thiebaut de Schotten M, Tomaiuolo F, Bartolomeo P (2008) White matter (dis)connections and gray matter (dys)functions in visual neglect: gaining insights into the brain networks of spatial awareness. Cortex 44:983-995.

Engel SA, Rumelhart DE, Wandell BA, Lee AT, Glover GH, Chichilnisky EJ, Shadlen MN (1994) fMRI of human visual cortex. Nature 369:525.

Fischl B, Sereno MI, Dale AM (1999) Cortical surface-based analysis. II: inflation, flattening, and a surface-based coordinate system. Neuroimage 9:195-207.

Fischl B, Liu A, Dale AM (2001) Automated manifold surgery: constructing geometrically accurate and topologically correct models of the human cerebral cortex. IEEE Trans Med Imaging 20:70-80.

Grefkes C, Ritzl A, Zilles K, Fink GR (2004) Human medial intraparietal cortex subserves visuomotor coordinate transformation. Neuroimage 23:1494-1506.

Harrison A, Jolicoeur P, Marois R (2010) "What" and "where" in the intraparietal sulcus: an fMRI study of object identity and location in visual short-term memory. Cereb Cortex. Advance online publication. Retrieved August 18, 2010. PMID: 20100899.

Heilman KM, Van Den Abell T (1980) Right hemisphere dominance for attention: the mechanism underlying hemispheric asymetries of inattention (neglect). Neurology 30:327-330.

Konen CS, Kastner S (2008a) Two hierarchically organized neural systems for object information in human visual cortex. Nat Neurosci 11:224-231.

Konen CS, Kastner S (2008b) Representation of eye movements and stimu- lus motion in topographically organized areas of human posterior parietal cortex. J Neurosci 28:8361-8375.

Luck SJ, Vogel EK (1997) The capacity of visual working memory for features and conjunctions. Nature 390:279-281.

Mesulam MM (1981) A cortical network for directed attention and unilateral neglect. Ann Neurol 10:309-325.

Mesulam MM (1999) Spatial attention and neglect: parietal, frontal and cingulate contributions to the mental representation and attentional targeting of salient extrapersonal events. Philos Trans R Soc Lond B Biol Sci 354:1325-1346.

Orban GA, Claeys K, Nelissen K, Smans R, Sunaert S, Todd JT, Wardak C, Durand JB, Vanduffel W (2006) Mapping the parietal cortex of human and non-human primates. Neuropsychologia 44:2647-2667.

Phillips WA (1974) On the distinction between sensory storage and shortterm visual memory. Percept Psychophys 16:283-290.

Pouget A, Driver J (2000) Relating unilateral neglect to the coding of space. Curr Opin Neurobiol 10:242-249.

Schluppeck D, Glimcher P, Heeger DJ (2005) Topographic organization for delayed saccades in human posterior parietal cortex. J Neurophysiol 94:1372-1384.

Sereno MI, Dale AM, Reppas JB, Kwong KK, Belliveau JW, Brady TJ, Rosen BR, Tootell RB (1995) Borders of multiple visual areas in humans revealed by functional magnetic resonance imaging. Science 268:889-893.

Sereno MI, Pitzalis S, Martinez A (2001) Mapping of contralateral space in retinotopic coordinates by a parietal cortical area in humans. Science 294:1350-1354.

Shulman GL, Pope DL, Astafiev SV, McAvoy MP, Snyder AZ, Corbetta M (2010) Right hemisphere dominance during spatial selective attention and target detection occurs outside the dorsal frontoparietal network. J Neurosci 30:3640-3651.

Silver MA, Kastner S (2009) Topographic maps in human frontal and parietal cortex. Trends Cogn Sci 13:488-495.

Silver MA, Ress D, Heeger DJ (2005) Topographic maps of visual spatial attention in human parietal cortex. J Neurophysiol 94:1358-1371.

Straw AD (2008) Vision egg: an open-source library for realtime visual stimulus generation. Front Neuroinformatics. 2:4

Swisher JD, Halko MA, Merabet LB, McMains SA, Somers DC (2007) Visual topography of human intraparietal sulcus. J Neurosci 27:5326-5337.

Szczepanski SM, Konen CS, Kastner S (2010) Mechanisms of spatial attention control in frontal and parietal cortex. J Neurosci 30:148-160.

Thesen S, Heid O, Mueller E, Schad LR (2000) Prospective acquisition correction for head motion with image-based tracking for real-time fMRI. Magn Reson Med 44:457-465.

Todd JJ, Marois R (2004) Capacity limit of visual short-term memory in human posterior parietal cortex. Nature 428:751-754.

Tootell RB, Hadjikhani N, Hall EK, Marrett S, Vanduffel W, Vaughan JT, Dale AM (1998) The retinotopy of visual spatial attention. Neuron 21:1409-1422.

Toth LJ, Assad JA (2002) Dynamic coding of behaviourally relevant stimuli in parietal cortex. Nature 415:165-168.

Vallar G, Perani D (1986) The anatomy of unilateral neglect after righthemisphere stroke lesions. A clinical/CT-scan correlation study in man. Neuropsychologia 24:609-622.

Vogel EK, Machizawa MG (2004) Neural activity predicts individual differences in visual working memory capacity. Nature 428:748-751.

Xu Y (2007) The role of the superior intraparietal sulcus in supporting visual short-term memory for multifeature objects. J Neurosci 27:11676-11686.

Xu Y, Chun MM (2006) Dissociable neural mechanisms supporting visual short-term memory for objects. Nature 440:91-95. 